RECENT ADVANCES

\title{
American fresh frozen plasma for neonates and children
}

\author{
D R Norfolk, A Glaser, S Kinsey
}

Arch Dis Child 2005;90:89-91. doi: 10.1136/adc.2003.048264

From the spring of 2004 the United Kingdom Blood Services have been importing fresh frozen plasma from United States donors for all neonates and children born after 1 January 1996. The decision to mandate the use of American plasma in this age group was taken by the Department of Health in 2002 as part of its precautionary approach to the risk of transfusion transmitted variant Creutzfeldt-Jakob disease. In this article we explain the background to this decision and explore some of the implications it raises for clinical practice.

See end of article for authors' affiliations $\ldots \ldots \ldots \ldots \ldots \ldots$

Correspondence to: Dr D R Norfolk, Department of Paediatric Oncology, Leeds Teaching Hospitals NHS Trust, Leeds LS9 7TF, UK; derek.norfolk@leedsth. nhs.uk

Accepted 23 May 2004 t is now generally agreed by experts that the epidemic of Creutzfeldt-Jakob disease (vCJD) in the United Kingdom is related to bovine spongiform encephalopathy (BSE) in cattle and was initially transmitted to humans by eating meat and other tissues from affected animals. As of 1 December 2003 there have been 143 cases of vCJD reported in the UK. Although the annual number of cases has fallen in the past three years, the scale of the final epidemic remains uncertain as the incubation period is long and may vary significantly between individuals depending on the route of transmission, age, and genetic susceptibility. ${ }^{1}$ It is assumed that the risk of acquiring vCJD from the food supply has fallen significantly since measures such as the Specified Bovine Materials Ban and Over Thirty Months rule were introduced and enhanced since 1996. Unlike classical CJD the abnormal prion of VCJD is widely distributed in haematological and lymphatic tissues. ${ }^{2}$ Its resistance to all known "pathogen inactivation" systems, combined with the lack of a screening test and our ignorance of the proportion of "healthy" blood donors incubating the disease, raises the spectre of an epidemic sustained by blood transfusion as the risk of food related transmission recedes. Animal experiments show that blood products from healthy donor animals in the incubation phase of the disease can transmit the infective prion. ${ }^{3}$

On 17 December 2003 the UK Secretary of State for Health made a Parliamentary statement giving details of the first probable case of vCJD due to transfused blood. The donor was healthy at the time of donation in March 1996 but presented with symptoms of the disease in 1999. The recipient of the blood developed clinical signs of vCJD within seven years and died in the autumn of 2003. Although it is possible that both donor and recipient acquired the disease by eating infected beef, there is a high probability that this is the first transfusion transmitted case, further suggested by the relatively short incubation period. ${ }^{4}$

WHAT HAS BEEN DONE ALREADY?

Although, until now, transfusion related vCJD has been only a theoretical risk, the UK government has applied the precautionary principle and instituted measures based on the assumption that transmission could occur. Since 1997 the Department of Health (DH) funded Transfusion Medicine Epidemiology Review has examined links between all vCJD cases and previous blood transfusion (the recent case was identified by this mechanism). All cases of confirmed or probable vCJD reported to the National CJD Surveillance Unit lead to a search of the National Blood Service (NBS) donor records. In total, 15 people in the UK have received blood from donors who subsequently developed vCJD. Since 1998 all blood donations have been leucodepleted by filtration soon after collection because of the suggestion that B lymphocytes are a particular source of abnormal prion and may be necessary for transfer of prions to the brain. However, evidence from the distribution of normal prion proteins in blood suggests that plasma and other blood components, such as platelets, are also likely to be sources of transmission. ${ }^{5}$ Since the end of 1999 all noncellular blood products used in the UK, except fresh frozen plasma (FFP) and cryoprecipitate, have been sourced from the USA and Germany, which are regarded as a low endemicity areas for BSE or vCJD. This includes products such as clotting factor concentrates, albumin, and immunoglobulins. UK citizens are now banned from donating blood in several western countries, and some countries have deferred donors from their own population who lived in the UK for extended periods between 1980 and 1996. The recent report of a single case of BSE in the northwestern USA is being followed with interest, although the policy to import US plasma remains unaltered at present.

\section{WHAT IS GOING TO HAPPEN?}

Following a recommendation from the Committee on the Microbiological Safety of Blood and Tissues (MSBT), the DH has instructed the NBS to switch to US sourced FFP for neonates and children born after 1 January 1996 as soon as a secure supply can be obtained. This patient group has been prioritised as they are considered

Abbreviations: BSE, bovine spongiform encephalopathy; $D H$, Department of Health; FFP, fresh frozen plasma; MB, methylene blue; NBS, National Blood Service; TRALI, transfusion related acute lung injury; VCJD, variant Creutzfeldt-Jakob disease 
at low risk of acquiring vCJD from food following the measures taken to keep infected material out of the human food chain. In December 2002 the DH purchased Life Resources Inc., the largest remaining independent US plasma collector, to ensure continuity of supply, and adequate supplies became available in spring 2004. The US donors are volunteer, unremunerated males (to reduce the risk of transfusion related acute lung injury, TRALI) and they will be tested for viral markers with the same stringency as UK donors. However, as there is a higher background level of viral markers in the US population, and to increase protection against emerging pathogens, it has been decided that the plasma must undergo a viral inactivation process (current UK FFP is "untreated"). To this end, the NBS has been developing systems for methylene blue plus light treatment of FFP (MB-FFP), and providing it for children and neonates since 2003. Before the switch to US FFP later this year, it is expected that the Chief Medical Officer for England will write to all paediatricians explaining the reasons for this change.

\section{WHAT ALTERNATIVES ARE AVAILABLE?}

Paediatricians will have two US sourced FFP products available. Firstly, single donor MB-FFP from the NBS. The $M B$ process uses photoinactivation to destroy lipid enveloped viruses and has been widely used in Europe for many years. The technique involves removal of most of the MB after inactivation and residual levels are believed to be well within safe limits for neonates and children. Secondly, a commercial pooled FFP product, Octaplas (produced by Octapharma), which uses the well tested solvent-detergent (SD) method to inactivate lipid enveloped viruses (SD-FFP). The decision as to which product to use is difficult and will depend, in the absence of comparative trials, on assumptions and perceptions about their potential risks and benefits. Key issues to consider include:

- MB-FFP is a single donor product (like currently available untreated FFP)

- Minimises donor exposure

- Effective against HIV, HBV, and HCV (one possible transmission reported)

- No effect on non-enveloped viruses such as parvovirus B19 or hepatitis A

- There is intrinsic variability in content of clotting factors (as with conventional FFP)

- Has significantly lower fibrinogen levels than untreated FFP and larger doses may be needed for therapeutic effect

- Small paediatric packs are available

- SD-FFP is produced from pooled donations (of up to 2500 donors)

- More potential donor exposures if process fails or nonsusceptible pathogen emerges (but excellent safety record in practice)

- Effective against HIV, HBV, and HCV

- No effect on non-enveloped viruses such as parvovirus B19 or hepatitis A

- Consistent, predictable clotting factor levels due to pooling

- Lower levels of protein S (may have contributed to cases of thrombosis in patients receiving large volumes for plasma exchange ${ }^{6}$ )

- Probable lower incidence of TRALI due to dilution of donor antibodies

- Not currently available in small paediatric packs.
Box 1: Clinical indications for the use of FFP in paediatrics*

- Children born after 1 January 1996 should only receive pathogen reduced FFP

- Bleeding due to haemorrhagic disease of the newborn: give FFP $10-20 \mathrm{ml} / \mathrm{kg}$ as well as intravenous vitamin $\mathrm{K}$ (grade C recommendation, level IV evidence)

- Neonates with coagulopathy who are bleeding, or are about to undergo an invasive procedure, should receive FFP and vitamin $\mathrm{K}$ (grade $\mathrm{C}$ recommendation, level IV evidence)

NB. Shortening of prolonged clotting times is unpredictable and should be checked following administration

- Routine administration of FFP to prevent periventricular haemorrhage in pre-term infants is not indicated (grade A recommendation, level Ilb evidence)

- FFP is not indicated in polycythaemia in infancy

- There are no definitive data to support clinical decisions about the use of FFP with low anti-T activity in neonates with T-activation (e.g. necrotising enterocolitis)

- FFP should only be used to replace single inherited clotting factor deficiencies for which no virus-safe fractionated product is available (currently factor V)

- FFP is indicated for demonstrable multi-factor deficiencies associated with severe bleeding and/or disseminated intravascular coagulation (DIC)

NB. FFP is not indicated in DIC with no evidence of bleeding and there is no evidence that prophylactic replacement prevents DIC or reduces transfusion requirements

${ }^{*}$ Adapted from BCSH Guidelines, $2004^{7}$

A much more detailed comparison is given in the recent Guidelines on FFP and Cryoprecipitate prepared by the British Committee for Standards in Haematology. ${ }^{7}$

\section{WHAT SHOULD WE DO?}

It is important that all clinicians involved in the management of neonates and children are aware of the issues discussed in this article. This is particularly important, as they will be in the front-line of implementing change and communicating with patients and parents. The decision as to which FFP product to use in individual patients is far from clear and will require discussion with local experts in transfusion medicine and other relevant specialties. There are no randomised, controlled trials to help us make this decision. We do know that FFP carries a higher risk of serious allergic and other immunological reactions than other blood products ${ }^{8}$ and that many transfusions are given for less than clear clinical indications. ${ }^{7}$ It is likely that the biggest single contribution to patient safety would be to restrict the use of FFP to evidence based indications wherever possible (box 1). We should be increasingly circumspect in the use of all blood products in neonates and young children ${ }^{9}$ as they have a lifetime in front of them to develop late complications of transfusion. Primum non nocere.

\section{Authors' affiliations}

D R Norfolk, A Glaser, S Kinsey, Leeds Teaching Hospitals NHS Trust, UK 


\section{REFERENCES}

1 Cooper JD, Bird SM. Predicting incidence of variant Creutzfeldt-Jakob disease from UK dietary exposure to bovine spongiform encephalopathy for the 1940 to 1969 and post-1969 birth cohorts. Int J Epidemiol 2003;32:784-91.

2 Hilton DA, Ghani AC, Conyers L, et al. Accumulation of abnormal prion protein in tonsil and appendix: review of tissue samples. BMJ 2002;325:633-4.

3 Hunter N, Foster J, Chong A, et al. Transmission of prion diseases by blood transfusion. J Gen Virol 2002;83:2897-905.

4 Bird SM. Recipients of blood or blood products "at vCJD risk". BMJ 2004;328:118-19.
5 MacGregor I, Hope J, Barnard G, et al. Application of a time-resolved fluoroimmunoassay for the analysis of normal prion protein in human blood and its components. Vox Sang 1999;77:88-96.

6 Yarranton H, Cohen H, Pavord SR, et al. Venous thromboembolism associated with the management of acute thrombotic thrombocytopenic purpura. Br J Haematol 2003;121:778-785.

7 British Committee for Standards in Haematology. www.bcshguidelines. com.

8 Serious Hazards of Transfusion (SHOT). Annual report 2002-2003, ISBN 095327895 6. www. shot-uk.org.

9 Murray NA, Roberts IAG. Neonatal transfusion practice. Arch Dis Child Fetal Neonatal Ed 2004;89:F101-7.
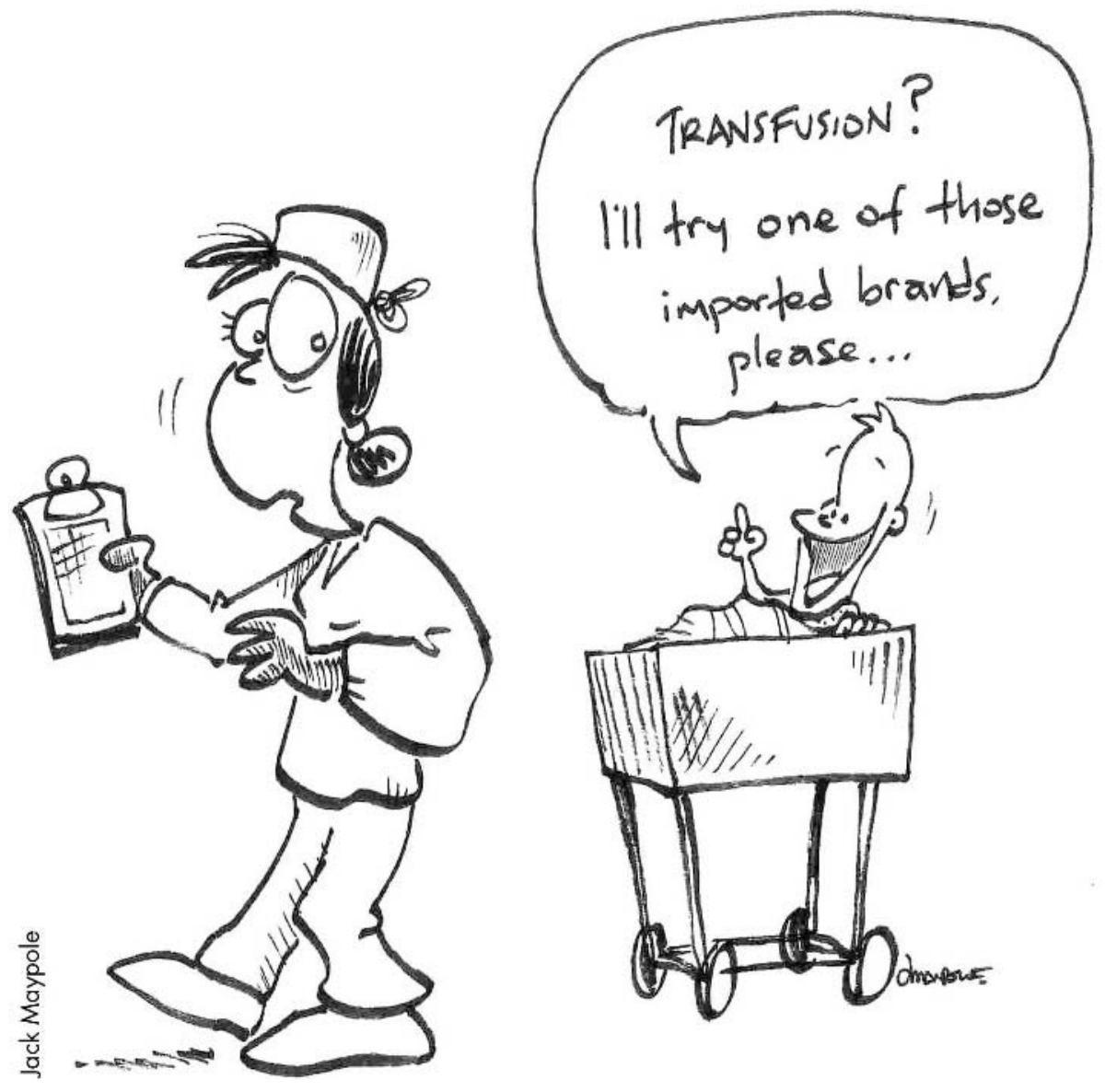